\title{
Normal radiological anatomy of thyroid cartilage in 600 Chinese individuals: implications for anterior cervical spine surgery
}

Ying-zhao Yan ${ }^{1,2,3}$, Chong-an Huang ${ }^{1}$, Qi Jiang ${ }^{4}$, Yi Yang ${ }^{3}$, Jian Lin ${ }^{1}, K$ Ke Wang ${ }^{1}$, Xiao-bin Li ${ }^{1}$, Hai-hua Zheng ${ }^{4^{*}}$ and Xiang-yang Wang ${ }^{1 *}$

\begin{abstract}
Background: Thyroid cartilage is an important barrier in anterior cervical approach surgery. The objective of this study is to establish normative values for thyroid cartilage at three planes and to determine their significance on preoperative positioning and intraoperative traction in surgery via the anterior cervical approach.

Methods: Neck CT scans were collected from 600 healthy adults who did not meet any of the exclusion criteria. Transverse diameters (D1, D2, and D3) of the superior border of the thyroid cartilage (SBTC), inferior border of the thyroid cartilage (IBTC), and the trachea transverse diameters of the inferior border of the cricoid cartilage (IBCC) were measured on a horizontal plane.

Results: All measured variables had intra-class correlation coefficients (ICCS) of $\geq 0.7$. The differences in transverse diameters on the same plane between males and females were significantly different (all $p<0.001$ ). The SBTC is most often at C4 in women (59.5\%) and C4/5 in men (36.4\%), the IBTC is most often at C5 in women (48.1\%) and men (46.2\%), and the IBCC is primarily located at C6 in women (45.2\%) and C6 or C6/7 in men (34.4\%) (all $p<0.001$ ).
\end{abstract}

Conclusion: We present normative values for thyroid cartilage at three planes of SBTC, IBTC, and IBCC in Chinese individuals. The individual and gender differences in the location of the thyroid cartilage and the size of the thyroid cartilage and the cricoid cartilage provide an anatomical basis to localize the skin incision, to predict the difficulty of intraoperative exposure and retractor pulling, and to identify that the thyroid cartilage protected the pharyngoesophageal wall.

Keywords: Thyroid cartilage, Cricoid cartilage, Anterior cervical spinal surgery, Anatomy, Positioning landmark, Pharyngoesophageal wall

\footnotetext{
*Correspondence: haihuazheng@wmu.edu.cn; xiangyangwang@wmu.edu.cn

Ying-Zhao Yan and Chong-an Huang should be considered as co-first authors.

Ying-Zhao Yan and Chong-an Huang contributed equally to this work.

${ }^{4}$ The Second Affiliated Hospital and Yuying Children's Hospital of Wenzhou Medical University, 109 Xueyuan Xi Road, Wenzhou, Zhejiang 325027, China

${ }^{1}$ Department of Spine Surgery, The Second Affiliated Hospital and Yuying

Children's Hospital of Wenzhou Medical University, Second Medical School of

Wenzhou Medical University, Zhejiang Spine Surgery Centre, Wenzhou,

Zhejiang 325027, China

Full list of author information is available at the end of the article
} 


\section{Background}

Anterior cervical (AC) surgery is associated with numerous and frequent complications that can be attributed to inaccurate positioning, inadequate exposure, and the use of excessive traction [1-3]. Attempts have been made to reduce the risk of complications in recent years. This has generally been achieved by improving body-surface positioning [1] and by evaluating the impact of the thyroid cartilage on intraoperative retraction $[4,5]$, as well as exploring hypopharynx/esophagus protective mechanisms.

Preoperative positioning in anterior cervical approach surgery generally utilizes anatomical structures such as the angle of the mandible, hyoid bone, thyroid cartilage, cricoid cartilage, and carotid artery nodule $[1,6]$. For example, approximately $70 \%$ of the mandibular angle is located at the interval between C2 and C3 [1], while the majority of cervical decompression surgeries are located below the C4 level [7]. Therefore, the angle of the mandible is not suitable for the majority of positions in anterior cervical approach surgery. The thyroid cartilage is closely associated with the cervical vertebra in terms of anatomy. It is located at the C4-C5 level, serves as a prominent anatomical landmark, and is therefore convenient for pre-surgical positioning in the anterior cervical approach.

Additionally, to expose the vertical vertebral level for surgery, significant retraction pressure may be exerted on the thyroid cartilage and posterior pharyngeal wall or esophagus. Wire retractor tension should thus be increased during surgery due to the great thyroid cartilage transverse diameter [4]. However, Han et al. [5] found that tension outside the thyroid cartilage would not increase the intra-esophageal pressure. Additionally, the posterior border of thyroid cartilage (PBTC) at the time of traction could protect the pharyngoesophageal (PE) wall [4]. However, such a standpoint requires further support from thyroid cartilage related-anatomical data.

Few studies in the literature have summarized the distribution of the transverse diameter of the superior and inferior border planes of the thyroid cartilage in addition to the relative cervical vertebra level $[8,9]$. This study aimed to measure the neck computed tomography (CT) images of healthy adults to obtain thyroid cartilage or cricoid cartilage imaging parameters to assist in preoperative positioning, to predict the difficulty of intraoperative exposure and retractor pulling, and to verify that the thyroid cartilage protected the PE wall of the anterior cervical approach.

\section{Methods}

\section{Subject enrollment and data collection}

This study received institutional review board approval and followed the principles of the Declaration of Helsinki. From January 2010 to September 2017, a total of 600 adults with healthy outpatient physical examination results at our hospital were enrolled for thyroid cartilage imaging measurements. Patients with no complaints of neck pain were included if they did not meet any of the exclusion criteria. The exclusion criteria were as follows: (1) diseases such as deformity, tuberculosis, or tumor of the larynx, thyroid cartilage, or thyroid, (2) diseases such as congenital deformity, torticollis, and neck stiffness in the neck structure, (3) spinal trauma, infection, tumor, or coronal deformities (Cobb angle > $10^{\circ}$ ) [10], (4) a history of prior spine surgery, (5) complaints of neck pain that affected activities of daily living, (6) degenerative or pathologic condition of the spine that necessitated physician intervention, (7) non-ambulatory patients, (8) pregnancy, and (9) inconsistent CT scan plane and measurement plane.

Patient height, weight and BMI were recorded after enrollment. All subjects underwent a $\mathrm{C} 1-\mathrm{T} 1$ thin-layer $\mathrm{CT}$ scan (layer thickness of 0.50 or $0.75 \mathrm{~mm}$ ) in our hospital.

\section{Radiographic analysis}

The thyroid cartilage imaging parameters of 600 healthy subjects were measured via a picture archiving and communication system with an accuracy of $0.01 \mathrm{~mm}$ under the bone window on the thin-layer CT cross section. The following radiographic parameters were measured: transverse diameters of the superior border of the thyroid cartilage (D1), transverse diameters of the inferior border of the thyroid cartilage (D2), and trachea transverse diameters of the inferior border of the cricoid cartilage (D3), as well as the transverse diameters of the corresponding vertebral body and intervertebral disc (V1, V2, and V3). Examples of D1, D2, D3, V1, V2, and $\mathrm{V} 3$ are shown in Fig. 1. The number of corresponding vertebral bodies or intervertebral discs on all planes was recorded.

The radiographs were reviewed by two authors who had independently measured more than several hundred cervical CT images prior to this review. The average of both reviewers' measurements was used for analysis.

\section{Statistical analyses}

The SPSS 19.0 statistical software package (SPSS Inc., Chicago, USA) was used for statistical analyses. Descriptive statistics including the mean, standard deviation, minimum, maximum, and percentiles were calculated for all variables. Measurement data conforming to a normal distribution were expressed as the mean \pm standard deviation.

The transverse diameters of the anatomical measurement data in all planes were compared between males and females using independent samples $t$ tests. The corresponding cervical level in all measurement planes was expressed as frequency and percentage and was compared 


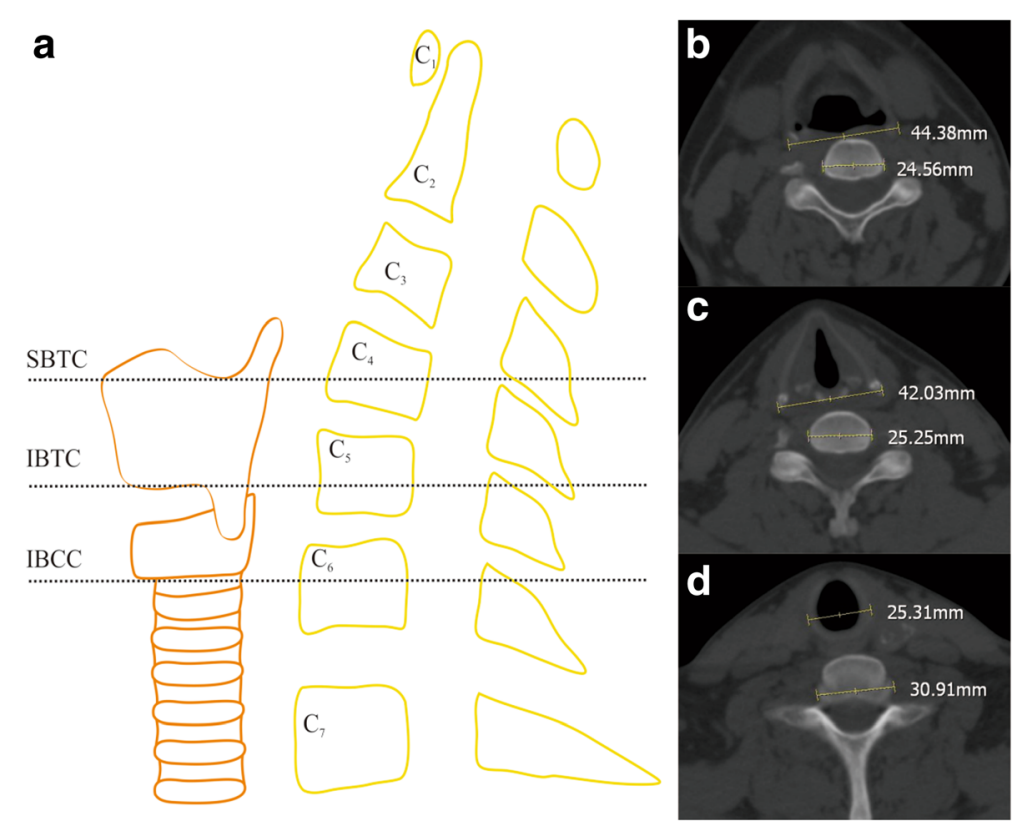

Fig. 1 The measurements used for this study. a Levels at which the thyroid and cricoid cartilage and cervical vertebrae were measured include the superior border of the thyroid cartilage (SBTC), the inferior border of the thyroid cartilage (IBTC), and the inferior border of the cricoids cartilage (IBCC). $\mathbf{b}$ The measurement of the SBTC. $\mathbf{c}$ The measurement of the IBTC. $\mathbf{d}$ The measurement of the IBCC

using a chi-squared test between males and females. $p$ values of less than 0.05 were considered significant.

A Pearson product-moment correlation coefficients table was then constructed to determine the correlations between all radiographic variables and age, weight, height, and BMI. Intra-class correlation coefficients (ICCs) and the kappa statistic were used for continuous and categorical intra-rater reliability data, respectively [9]. The internal consistency of the measurements was characterized as excellent $(\geq 0.9)$, good $(\geq 0.7$ and $<0.9)$, acceptable $(\geq 0.6$ and $<0.7)$, poor $(\geq 0.5$ and $<0.6)$, or unpredictable $(<0.5)$ [10].

\section{Results}

\section{Patient characteristics}

There were 600 healthy study subjects aged $54.1 \pm$ 10.0 years (range 24 to 77), with a height of $165.0 \pm$ $7.2 \mathrm{~cm}$ (range 145 to 180), weight of $63.3 \pm 10.1 \mathrm{~kg}$ (range 41 to 90 ), and BMI of $23.1 \pm 2.8 \mathrm{~kg} / \mathrm{m}^{2}$ (range 16.9 to 31.8 ). Overall, 390 subjects were males aged 24 to 77 years, with a height of 145 to $180 \mathrm{~cm}$, weight of 49 to $90 \mathrm{~kg}$, and BMI of 18.4 to $31.1 \mathrm{~kg} / \mathrm{m}^{2}$. A total of 210 subjects were females aged 31 to 74 years, with a height of 148 to $173 \mathrm{~cm}$, weight of 41 to $81.5 \mathrm{~kg}$, and BMI of 16.9 to $31.8 \mathrm{~kg} / \mathrm{m}^{2}$.

Because we intended to establish normative values based on gender, effort was made to maintain an even distribution of age and BMI between males and females. The demographic data are included in Table 1. The differences in age and BMI between males and females were not significant $(p>0.05)$.

\section{Radiographic results}

Overall, we had good (ICC or Kappa $\geq 0.7$ ) to excellent inter-observer reliability for all included radiographic measurements (Table 2); the majority of measurements exhibited excellent reliability.

The average values for each variable, including the mean, standard deviation range, and percentiles, are reported in Table 3 . The average values by gender are reported in Table 4. The transverse diameters of the thyroid cartilage or trachea (D1, D2, and D3) gradually decreased in all the planes from top to bottom, while those of the vertebral body or intervertebral disc (V1, V2, and V3) gradually increased. Differences on the same plane between males and females were significant (all $p<0.001$ ).

When examining the change in alignment with age, we found that V1 $(r=0.124, p<0.05)$, V2 $(r=0.202, p$ $<0.01)$, and V3 $(r=0.130, p<0.01)$ all increased with age (Table 5 ). There was no correlation between age and

Table 1 Demographic data of healthy adults

\begin{tabular}{lllll}
\hline & Total & Male & Female & $p$ value \\
\hline Number of cases & 600 & 390 & 210 & - \\
Age (years) & $54.1 \pm 10.0$ & $53.7 \pm 9.4$ & $55.1 \pm 10.9$ & 0.106 \\
Weight $(\mathrm{kg})$ & $63.3 \pm 10.1$ & $66.4 \pm 9.1$ & $57.5 \pm 9.1$ & 0.000 \\
Height $(\mathrm{cm})$ & $165.0 \pm 7.2$ & $168.6 \pm 5.2$ & $158.4 \pm 5.6$ & 0.000 \\
BMl $\left(\mathrm{kg} / \mathrm{m}^{2}\right)$ & $23.1 \pm 2.8$ & $23.3 \pm 2.6$ & $22.9 \pm 3.0$ & 0.069 \\
\hline
\end{tabular}

Data presented as the mean \pm SD

The significant difference was compared between males and females 
Table 2 Measurement reliability statistics for various radiographic values

\begin{tabular}{|c|c|c|c|}
\hline & Kappa & ICC & Inter-rater reliability \\
\hline CLCEP & 0.9 & & Excellent \\
\hline Transverse diameter of thyroid cartilage of SBTC (D1) & & 0.8 & Good \\
\hline Transverse diameter of vertebral body or intervertebral disc of SBTC (V1) & & 0.8 & Good \\
\hline Transverse diameter of thyroid cartilage of IBTC (D2) & & 0.9 & Excellent \\
\hline Transverse diameter of vertebral body or intervertebral disc of IBTC (V2) & & 0.7 & Good \\
\hline Transverse diameter of trachea of IBCC (D3) & & 0.9 & Excellent \\
\hline Transverse diameter of vertebral body or intervertebral disc of IBCC (V3) & & 0.9 & Excellent \\
\hline
\end{tabular}

CLCEP cervical level corresponding to each plane, SBTC plane of superior border of the thyroid cartilage, IBTC plane of inferior border of the thyroid cartilage, IBCC plane of inferior border of the cricoid cartilage, ICC intra-class correlation coefficient

D1, D2, and D3. However, a higher BMI was correlated to an increase in $\mathrm{D} 1(r=0.148, p<0.01)$, D2 $(r=0.136$, $p<0.01)$, and $\mathrm{D} 3(r=0.215, p<0.01)$. Interestingly, increasing weight and height were correlated to increasing $\mathrm{D} 1, \mathrm{D} 2, \mathrm{D} 3$, and V1, V2, and V3 (all $p<0.01$ ). These correlations are detailed in Table 5 .

The differences in the distribution of the corresponding cervical levels on SBTC, IBTC, and IBCC are reported in Fig. 2. The distribution was also significant between males and females $(p<0.001$, Table 6). The SBTC corresponded to levels C3/4 to C6 and was mainly located at C4 $(n=263)$, accounting for $43.8 \%$ of cases, while $560(93.3 \%)$ were located at level C4 to C5. The IBTC corresponded to levels $\mathrm{C} 4$ to $\mathrm{C} 7$, with the majority located at C5 $(n=281)$, accounting for $46.8 \%$ of cases. Meanwhile, $530(88.3 \%)$ were located at levels $C 4 / 5$ to C5/6. The IBCC corresponded to levels C5 to T1, with most located at C6 $(n=229)$, accounting for $38.2 \%$ of cases. A total of $556(92.7 \%)$ cases were located at levels C5/6 to C7.

\section{Discussion}

In this study, we have presented normative values for D1, D2, and D3 as well as V1, V2, and V3. To the best of our knowledge, these values have not been reported in any previous studies. In addition, we found that SBTC is most often at C4 in women and C4/5 in men, IBTC is most often at $\mathrm{C} 5$ in women and men, and IBCC is most

Table 3 Average values and percentiles for all measured radiographic parameters

\begin{tabular}{|c|c|c|c|c|c|c|c|c|}
\hline & \multirow{2}{*}{$\begin{array}{l}\text { Mean } \\
(\mathrm{mm})\end{array}$} & \multirow{2}{*}{$\begin{array}{l}\text { Min } \\
(\mathrm{mm})\end{array}$} & \multirow{2}{*}{$\begin{array}{l}\text { Max } \\
(\mathrm{mm})\end{array}$} & \multicolumn{5}{|c|}{ Percentiles } \\
\hline & & & & 5 & 25 & 50 & 75 & 95 \\
\hline D1 & $44.4 \pm 5.0$ & 34.0 & 60.0 & 36.1 & 41.1 & 44.0 & 47.3 & 53.2 \\
\hline D2 & $41.5 \pm 4.6$ & 31.2 & 54.5 & 34.3 & 38.3 & 41.3 & 45.1 & 49.4 \\
\hline D3 & $26.5 \pm 3.8$ & 18.8 & 34.5 & 20.4 & 23.2 & 27.1 & 29.3 & 32.6 \\
\hline V1 & $27.5 \pm 2.6$ & 21.4 & 35.5 & 23.1 & 25.9 & 27.4 & 29.0 & 31.9 \\
\hline V2 & $29.1 \pm 3.2$ & 16.2 & 40.0 & 24.8 & 27.1 & 28.5 & 30.4 & 35.9 \\
\hline V3 & $31.6 \pm 3.4$ & 24.1 & 41.0 & 26.4 & 29.1 & 31.6 & 33.7 & 37.3 \\
\hline
\end{tabular}

often at C6 in women and C6 or C6/7 in men in Chinese (Table 6). The results of our work are generally in good agreement with previous studies $[1,9,11]$. Contemporary anatomy texts indicate that the upper border of the thyroid cartilage is at C3/4 or C4 in the USA [11]. A more recent CT study of over 52 asymptomatic New Zealanders found that the vertebral level corresponding to the upper border of the thyroid cartilage was sexually dimorphic and was most often at C4 in women and C5 in men. The inferior border of the cricoid cartilage was also significantly different in women and men (C6 and C7, respectively) [9]. Additionally, we identified changes in these parameters with age, weight, height, and BMI (Table 5). We found that V1, V2, and V3 all increased with age. This may be due to the development and calcification of thyroid cartilage [12, 13]. However, it is curious that a higher BMI was correlated to an increase in D1, D2 and D3 but not correlated to V1, V2, and V3. We were also able to show that weight and height were correlated to increasing D1, D2, D3 and V1, V2, V3. This finding is not surprising given the increase in bone size with height and weight in the normal BMI range. However, the correlation coefficient between D1, D2, D3, and weight and height is higher than that between V1, V2, V3, and weight and height.

There is general agreement on the vertebral levels of key palpable landmarks in anterior cervical spine surgery.

Table 4 Radiographic parameters by gender

\begin{tabular}{|c|c|c|c|}
\hline & Male (mm) & Female $(\mathrm{mm})$ & $p$ value \\
\hline D1 & $46.6 \pm 4.5$ & $40.4 \pm 2.8$ & 0.000 \\
\hline D2 & $43.7 \pm 3.7$ & $37.3 \pm 2.8$ & 0.000 \\
\hline D3 & $28.7 \pm 2.5$ & $22.4 \pm 1.7$ & 0.000 \\
\hline V1 & $28.4 \pm 2.5$ & $26.0 \pm 2.1$ & 0.000 \\
\hline V2 & $30.0 \pm 3.2$ & $27.3 \pm 2.1$ & 0.000 \\
\hline V3 & $32.9 \pm 3.0$ & $29.0 \pm 2.4$ & 0.000 \\
\hline
\end{tabular}


Table 5 Correlations of all radiographic parameters with age, weight, height, and BMI

\begin{tabular}{lllll}
\hline & Age & Weight & Height & BMl \\
\hline D1 & -.047 & $.400^{\mathrm{a}}$ & $.527^{\mathrm{a}}$ & $.148^{\mathrm{a}}$ \\
V1 & $.124^{\mathrm{b}}$ & $.244^{\mathrm{a}}$ & $.350^{\mathrm{a}}$ & .073 \\
D2 & -.073 & $.394^{\mathrm{a}}$ & $.531^{\mathrm{a}}$ & $.136^{\mathrm{a}}$ \\
V2 & $.202^{\mathrm{a}}$ & $.163^{\mathrm{a}}$ & $.331^{\mathrm{a}}$ & -.025 \\
D3 & .019 & $.505^{\mathrm{a}}$ & $.626^{\mathrm{a}}$ & $.215^{\mathrm{a}}$ \\
V3 & $.130^{\mathrm{a}}$ & $.187^{\mathrm{a}}$ & $.430^{\mathrm{a}}$ & -.064 \\
\hline
\end{tabular}

$D 1, D 2$, and $D 3$ transverse diameter of thyroid cartilage or trachea of SBTC, IBTC, and IBCC

$V_{1}, V_{2}$, and $V_{3}$ transverse diameter of vertebral body or intervertebral disc of SBTC, IBTC, and IBCC

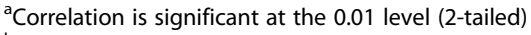

${ }^{\mathrm{b}}$ Correlation is significant at the 0.05 level (2-tailed)

However, inaccurate preoperative skin positioning may lead to an enlarged intraoperative incision and range of exposure, resulting in an increase in both the operation time and tension of the intraoperative soft tissue traction [1]. Therefore, an appropriate skin incision can maximize the surgical field and avoid expanding the incision, which may otherwise affect the esthetics and increase the extent of the stripping, thus leading to an increased risk of postoperative anterior cervical hematoma and dysphagia due to elevated hemorrhage and continuous traction [3]. Kirschner wire and other fine metals are extensively used as a reference prior to skin cutting and decompression in anterior cervical approach surgery followed by $\mathrm{C}$-arm perspective positioning. However, an acquaintance with the related anatomical positioning markers close to the cervical level contributes to a reduction in repeated perspective adjustments, thus saving a great deal of time and avoiding an increase in radiation exposure.

Preoperative positioning in anterior cervical approach surgery generally utilizes anatomical structures such as the angle of the mandible, hyoid bone, thyroid cartilage, cricoid cartilage, and carotid artery nodule [1, 6]. The mandibular angle is located at the interval between $\mathrm{C} 2$ and C3 [1], while the majority of cervical decompression surgeries are located below the C4 level [7]. Therefore, the angle of the mandible is not suitable, although the distance from it to the surgical level can be measured on preoperative X-ray films. The thyroid cartilage, however, is mostly located at the C4-C5 level according to the present study and is more or less fixed in front of the cervical vertebrae apart from when swallowing or intonation occurs. The cartilage does not easily move with body position but is easily accessible compared with the hyoid bone and carotid artery nodule. Additionally, the anatomical location of the thyroid cartilage angle is more obvious in men due to the presence of the Adam's apple. Therefore, preoperative X-rays allow for the location of almost all anterior cervical approach surgical levels.

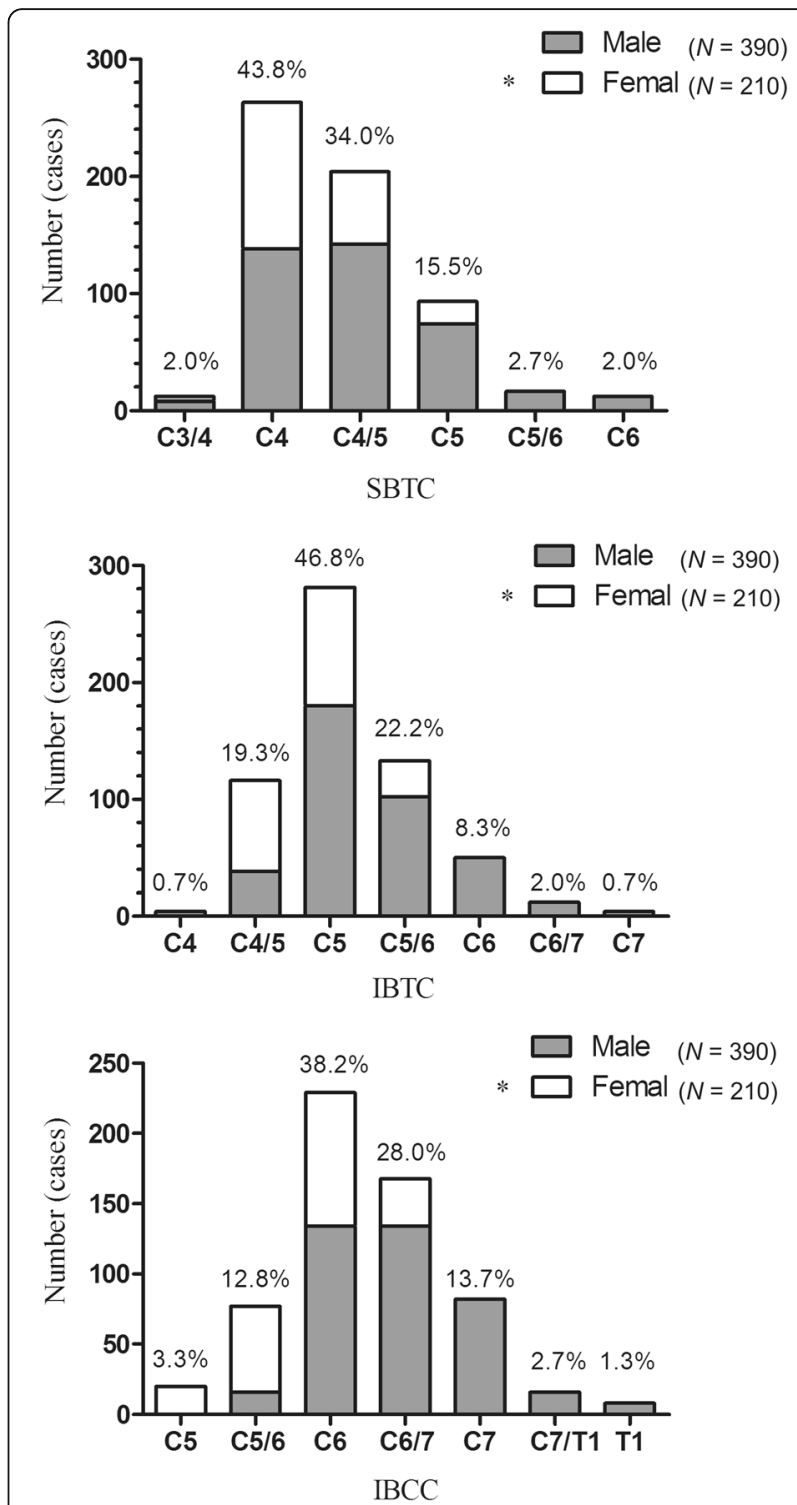

Fig. 2 The frequency distribution of the cervical vertebral level corresponding to SBTC, IBTC, and IBCC by gender. SBTC superior border of the thyroid cartilage, IBTC inferior border of the thyroid cartilage, IBCC inferior border of the cricoids cartilage. ${ }^{*} p<0.001$, compared with males

Therefore, the corresponding surgical level can be judged rapidly based on the position of the SBTC, IBTC, and IBCC prior to skin cutting and decompression.

The thyroid cartilage or trachea and the posterior pharyngeal wall or esophagus in front of the surgical level should be pulled to one side during surgery. Therefore, a wide thyroid cartilage will often add to the difficulties incurred in exposing the surgical level. The thyroid cartilage is an important barrier in anterior cervical approach surgery. Chio et al. [4] discovered through intraoperative ultrasound positioning that the thyroid cartilage rarely rotated along with the traction of the retractor, while 
Table 6 The frequency distribution of cervical segments corresponding to each plane in 600 healthy subjects

\begin{tabular}{|c|c|c|c|c|c|c|c|c|c|}
\hline \multirow[t]{2}{*}{ Level } & \multicolumn{3}{|l|}{ SBTC } & \multicolumn{3}{|l|}{ IBTC } & \multicolumn{3}{|l|}{ IBCC } \\
\hline & $\overline{\text { Male }^{*}(\%)}$ & Female (\%) & All (\%) & Male $^{*}(\%)$ & Female (\%) & All (\%) & Male $^{*}(\%)$ & Female (\%) & All (\%) \\
\hline $\mathrm{C} 3 / 4$ & $8(2.1)$ & $4(1.9)$ & $12(2.0)$ & 0 & 0 & 0 & 0 & 0 & 0 \\
\hline C4 & $138(35.4)$ & $125(59.5)$ & $263(43.8)$ & $4(1.0)$ & 0 & $4(0.7)$ & 0 & 0 & 0 \\
\hline$C 4 / 5$ & $142(36.4)$ & $62(29.5)$ & $204(34.0)$ & $38(9.7)$ & 78 (37.1) & $116(19.3)$ & 0 & 0 & 0 \\
\hline C5 & 74 (19.0) & $19(9.0)$ & $93(15.5)$ & $180(46.2)$ & $101(48.1)$ & $281(46.8)$ & 0 & $20(9.5)$ & $20(3.3)$ \\
\hline C5/6 & $16(4.1)$ & 0 & $16(2.7)$ & $102(26.2)$ & $31(14.8)$ & $133(22.2)$ & $16(4.1)$ & 61 (29.0) & 77 (12.8) \\
\hline C6 & $12(3.1)$ & 0 & $12(2.0)$ & $50(12.8)$ & 0 & $50(8.3)$ & $134(34.4)$ & $95(45.2)$ & $229(38.2)$ \\
\hline $\mathrm{C} 6 / 7$ & 0 & 0 & 0 & $12(3.1)$ & 0 & $12(2.0)$ & $134(34.4)$ & $34(16.2)$ & $168(28.0)$ \\
\hline$C 7$ & 0 & 0 & 0 & $4(1.0)$ & 0 & $4(0.7)$ & $82(21.0)$ & 0 & 82 (13.7) \\
\hline $\mathrm{C} 7 / \mathrm{T} 1$ & 0 & 0 & 0 & 0 & 0 & 0 & $16(4.1)$ & 0 & $16(2.7)$ \\
\hline $\mathrm{T} 1$ & 0 & 0 & 0 & 0 & 0 & 0 & $8(2.1)$ & 0 & $8(1.3)$ \\
\hline Total & $390(100)$ & $210(100)$ & 600 (100) & 390 (100) & 210 (100) & 600 (100) & $390(100)$ & $210(100)$ & $600(100)$ \\
\hline
\end{tabular}

Percentages are shown in brackets

SBTC superior border of the thyroid cartilage, IBTC inferior border of the thyroid cartilage, IBCC inferior border of the cricoid cartilage ${ }^{*} p<0.001$ for males versus females using the chi-squared test

translation of the thyroid cartilage with the posterior pharyngoesophagus was common. The traction of the retractor should be increased when pulling a larger thyroid cartilage to sufficiently expose the surgical level. Nevertheless, increasing intraoperative traction may lead to cervical hematoma and soft tissue swelling. However, Han et al. [5] conducted a study in 2015 in which the tension of the retractor at the time of pulling and the pressure in the esophagus were measured. Moreover, it was verified that increasing the tension outside the thyroid cartilage would not increase the pressure in the esophagus. In contrast, translation of a larger thyroid cartilage with the posterior pharyngoesophagus can be seen at the time of traction, which can protect the PE wall [4].

The exact pathogenesis of cervical esophageal injury remains unknown [7]. Although the incidence of PE injury has steadily increased [14-16], PE injury is unfortunately hard to diagnose even with advancements in radiography [17]. Thus, the prevention of injury is most important during anterior cervical spine surgery. Direct injury with an instrument or retractor during dissection is a possible cause of PE injury $[15,18,19]$. Our results (Tables 3 and 4) suggest that the average transverse diameter of the SBTC and IBTC or IVCC gradually decreased in healthy adults from top to bottom, while that of the corresponding cervical vertebral body or intervertebral disc gradually increased. Therefore, the traction of the retractor should be increased when conducting highlevel anterior cervical approach surgery to sufficiently expose the surgical level, which will therefore lead to cervical hematoma formation and soft-tissue swelling [3]. In contrast, the required traction of the retractor when performing lower cervical surgery is small since the coverage width of the thyroid cartilage gradually decreases; however, this may lead to direct pressure being placed on the esophagus. According to previous studies $[15,16], \mathrm{PE}$ wall injury most often appears in lower cervical surgery $(\mathrm{C} 5-\mathrm{C} 7)$, which may be related to a susceptibility to direct intraoperative traction $[18,19]$. The anatomical location of the thyroid cartilage was further identified in this study, and it was found that the thyroid cartilage was mostly located above the C5 level (66.8\%), while the trachea was mostly located below the C5 level. Such an anatomical feature provides a basis for the view that the thyroid cartilage does not protect the PE wall in lower cervical surgery (C5-C7). Therefore, when operating below the $\mathrm{C} 5$, special care should be taken during retraction to prevent injury of the esophagus wall.

This study does, however, acknowledge some weaknesses. Since this is a retrospective study, it is not possible to evaluate the patient face to face. Our sample size is considerable compared to other similar studies $[8,9]$. The selection of individuals was only based on patient complaints and history records, and it is possible for these data to be skewed by outliers. A second limitation of our study is the relatively high proportion of elderly individuals enrolled. This occurred because age was not used as a screening criterion when cases were selected. While the aged-predominant nature of the population might limit our ability to draw conclusions about age variability, we do not believe it will limit the relevance of our work to anterior cervical spine surgery. Standardized head positions in CT scans are also different among different studies [9], but we have excluded patients with CT scan planes that are not consistent with the measurement plane because of incorrect head positions. Finally, it is important to acknowledge that the population in this study is drawn from an urban, university hospital 
in East China. Surgeons whose practice setting is significantly different from ours must be aware of the potential for ethnic- and race-based differences in normative values.

\section{Conclusions}

In summary, we present normative values for the thyroid cartilage and cricoid cartilage at three planes of SBTC, IBTC, and IBCC in Chinese individuals. The individual and gender differences in the location of the thyroid cartilage and the size of the thyroid cartilage and the cricoid cartilage provided an anatomical basis to help localize the skin incision, to predict the difficulty of intraoperative exposure and retractor pulling, and to verify that the thyroid cartilage protects the PE wall.

\section{Abbreviations \\ AC: Anterior cervical; ACDF: Anterior cervical discectomy and fusion; D1, D2, D3: Transverse diameter of thyroid cartilage or trachea of SBTC, IBTC, and IBCC; IBCC: Inferior border of the cricoid cartilage; IBTC: Inferior border of the thyroid cartilage; ICC: Intra-class correlation coefficient; PBTC: Posterior border of thyroid cartilage; PE: Pharyngoesophageal; SBTC: Superior border of the thyroid cartilage; V1, V2, V3: Transverse diameter of vertebral body or intervertebral disC of SBTC, IBTC, and IBCC}

\section{Acknowledgements}

We would like to thank Ai-min Wu, MD, PhD, The Second Affiliated Hospital and Yuying Children's Hospital of Wenzhou Medical University, for his thoughtful discussions.

\section{Funding}

This work is supported by a grant from the National Nature Foundation of China (Grant No. 81371988) and a major science and technology program for medical and health of Zhejiang Province (Grant No.WKJ-ZJ-1527).

\section{Availability of data and materials}

We state that the data will not be shared since all raw data used are presented in the figures that are included in the article.

\section{Authors' contributions}

$Y Z Y, X Y W$, and $H H Z$ designed the study. QJ, JL, XBL, and KW collected the data. CAH and YZY performed the statistical analysis and interpreted the results. CAH, YZY, QJ, and YY drafted the manuscript. All of the authors critically revised the manuscript. The final manuscript was approved by all authors.

\section{Ethics approval and consent to participate}

This study was approved by the IRB (Institutional Review Board) of the Second Affiliated Hospital and Yuying Children's Hospital of Wenzhou Medical University. Patient's consent to participate was not required in this retrospective study.

\section{Consent for publication}

Not applicable.

\section{Competing interests}

The authors declare that they have no competing interests.

\section{Publisher's Note}

Springer Nature remains neutral with regard to jurisdictional claims in published maps and institutional affiliations.

\section{Author details}

'Department of Spine Surgery, The Second Affiliated Hospital and Yuying Children's Hospital of Wenzhou Medical University, Second Medical School of Wenzhou Medical University, Zhejiang Spine Surgery Centre, Wenzhou,
Zhejiang 325027, China. ${ }^{2}$ Department of Orthopaedic Surgery, The Fifth Affiliated Hospital of Wenzhou Medical University, Lishui Central Hospital, Lishui, Zhejiang 323000, China. ${ }^{3}$ Department of Internal Medicine, The Sixth Affiliated Hospital of Wenzhou Medical University, The People's Hospital of Lishui, Lishui, Zhejiang 323000, China. ${ }^{4}$ The Second Affiliated Hospital and Yuying Children's Hospital of Wenzhou Medical University, 109 Xueyuan Xi Road, Wenzhou, Zhejiang 325027, China.

Received: 25 October 2017 Accepted: 23 January 2018

Published online: 08 February 2018

\section{References}

1. Auerbach JD, Weidner Z, Pill SG, Mehta S, Chin KR. The mandibular angle as a landmark for identification of cervical spinal level. Spine (Phila Pa 1976). 2009:34(10):1006-11. https://doi.org/10.1097/BRS.0b013e31819f2a03.

2. Fountas KN, Kapsalaki EZ, Nikolakakos LG, Smisson HF, Johnston KW, Grigorian AA, Lee GP, Jr RJ. Anterior cervical discectomy and fusion associated complications. Spine (Phila Pa 1976). 2007;32(21):2310-7. https:// doi.org/10.1097/BRS.0b013e318154c57e.

3. Kepler CK, Rihn JA, Bennett JD, Anderson DG, Vaccaro AR, Albert TJ, Hilibrand AS. Dysphagia and soft-tissue swelling after anterior cervical surgery: a radiographic analysis. Spine J. 2012;12(8):639-44. https://doi.org/ 10.1016/j.spinee.2012.03.024.

4. Choi BK, Cho WH, Choi CH, Song GS, Kim C, Kim HJ. Hypopharyngeal wall exposure within the surgical field : the role of axial rotation of the thyroid cartilage during anterior cervical surgery. J Korean Neurosurg Soc. 2010; 48(5):406-11. https://doi.org/10.3340/jkns.2010.48.5.406.

5. Han IH, Lee SH, Lee JM, Kim HS, Nam KH, Duetzmann S, Park J, Choi BK. Does a medial retraction blade transmit direct pressure to pharyngeal/ esophageal wall during anterior cervical surgery? Spine (Phila Pa 1976). 2015;40(1):E18-22. https://doi.org/10.1097/BRS.0000000000000649.

6. Reinard KA, Cook DM, Zakaria HM, Basheer AM, Chang WW, Abdulhak MM. A cohort study of the morbidity of combined anterior-posterior cervical spinal fusions: incidence and predictors of postoperative dysphagia. Eur Spine J. 2016 Jul;25(7):2068-77. https://doi.org/10.1007/s00586-016-4429-0.

7. Konstantakos AK, Temes RT. Delayed esophageal perforation: a complication of anterior cervical spine fixation. Ann Thorac Surg. 2005;80(1):349. https:// doi.org/10.1016/50003-4975(03)01370-5.

8. Ozguner G, Sulak O. Size and location of thyroid gland in the fetal period. Surg Radiol Anat. 2014;36(4):359-67. https://doi.org/10.1007/s00276-013-1177-2.

9. Mirjalili SA, Mcfadden SL, Buckenham T, Stringer MD. Vertebral levels of key landmarks in the neck. Clin Anat. 2012;25(7):851-7. https://doi.org/10.1002/ ca.22124.

10. Iyer S, Lenke LG, Nemani VM, Fu M, Shifflett GD, Albert TJ, Sides BA, Metz LN, Cunningham ME, Kim HJ. Variations in occipitocervical and cervicothoracic alignment parameters based on age: a prospective study of asymptomatic volunteers using full-body radiographs. Spine (Phila Pa 1976). 2016;41(23):1837-44. https://doi.org/10.1097/BRS.0000000000001644.

11. Drake RL, Vogl AW, Mitchell AWM. Gray's anatomy for students. 2nd ed. Philadelphia: Churchill Livingstone; 2010. p. 803, 806, 1063.

12. Glikson E, Sagiv D, Eyal A, Wolf M, Primov-Fever A. The anatomical evolution of the thyroid cartilage from childhood to adulthood: a computed tomography evaluation. Laryngoscope. 2017;127(10):E354-8. https://doi.org/ 10.1002/lary.26644.

13. Wenaas AE, Tran B, Ongkasuwan J. The progression of thyroid cartilage calcification as it relates to the utilization of laryngeal ultrasound. Laryngoscope. 2016;126(4):913-7. https://doi.org/10.1002/lary.25582.

14. Orlando ER, Caroli E, Ferrante L. Management of the cervical esophagus and hypofarinx perforations complicating anterior cervical spine surgery. Spine (Phila Pa 1976). 2003;28(15):E290-5. https://doi.org/10.1097/01.BRS. 0000087093.89889.0A

15. Patel NP, Wolcott WP, Johnson JP. Esophageal injury associated with anterior cervical spine surgery. Surg Neurol. 2008;69(1):20-4. https://doi.org/ 10.1016/j.surneu.2007.05.006.

16. Nam KH, Kim DH, Kim HS, Han IH, Shin N, Choi BK. Cadaveric analysis of posterior pharyngoesophageal wall thickness: Implicationsfor anterior cervical spine surgery. Spine (Phila Pa 1976). 2016;41(5):E262-7. https://doi. org/10.1097/BRS.0000000000001217.

17. Taylor B, Patel AA, Okubadejo GO, Albert T, Riew KD. Detection of esophageal perforation using intraesophageal dye injection. J Spinal Disord Tech. 2006;19(3):191-3. https://doi.org/10.1097/01.bsd.0000190559.20872.2a. 
18. Zhong ZM, Jiang JM, Qu DB, Wang J, Li XP, Lu KW, Xu B, Chen JT. Esophageal perforation related to anterior cervical spinal surgery. J Clin Neurosci. 2013;20(10):1402-5. https://doi.org/10.1016/j.jocn.2012.11.020.

19. Gaudinez RF, English GM, Gebhard JS, Brugman JL, Donaldson DH, Brown CW. Esophageal perforations after anterior cervical surgery. J Spinal Disord. 2000;13(1):77-84. PMID: 10710155

Submit your next manuscript to BioMed Central and we will help you at every step:

- We accept pre-submission inquiries

- Our selector tool helps you to find the most relevant journal

- We provide round the clock customer support

- Convenient online submission

- Thorough peer review

- Inclusion in PubMed and all major indexing services

- Maximum visibility for your research

Submit your manuscript at www.biomedcentral.com/submit 